\title{
Quantifying the anomalous self-diffusion in molybdenum with first-principles simulations
}

\author{
Thomas R. Mattsson, Nils Sandberg, Rickard Armiento and Ann E. Mattsson
}

\section{Post Print}

N.B.: When citing this work, cite the original article.

Original Publication:

Thomas R. Mattsson, Nils Sandberg, Rickard Armiento and Ann E. Mattsson, Quantifying the anomalous self-diffusion in molybdenum with first-principles simulations, 2009, Physical Review B. Condensed Matter and Materials Physics, (80), 22, 224104.

http://dx.doi.org/10.1103/PhysRevB.80.224104

Copyright: American Physical Society http://www.aps.org/

Postprint available at: Linköping University Electronic Press http://urn.kb.se/resolve?urn=urn:nbn:se:liu:diva-86289 


\title{
Quantifying the anomalous self-diffusion in molybdenum with first-principles simulations
}

\author{
T. R. Mattsson \\ HEDP Theory, Sandia National Laboratories, Albuquerque, New Mexico 87185-1189, USA \\ N. Sandberg \\ Department of Physics, Royal Institute of Technology, Stockholm SE-100 44, Sweden \\ R. Armiento \\ Theoretische Physik, Universität Bayreuth, D-95440 Bayreuth, Germany
}

\author{
A. E. Mattsson \\ Multiscale Dynamic Materials Modeling, Sandia National Laboratories, Albuquerque, New Mexico 87185-1322, USA \\ (Received 8 October 2009; revised manuscript received 28 October 2009; published 14 December 2009)
}

\begin{abstract}
First-principles molecular-dynamics simulations based on a recently developed exchange-correlation functional show that self-diffusion in the refractory metal molybdenum is associated with strongly temperaturedependent activation energies for vacancy formation and migration. While static calculations of self-diffusion rates based on transition-state theory deviate systematically from experiments, with up to two orders of magnitude, the current results are accurate to within a mean deviation of 4 over the experimental range in temperature.
\end{abstract}

DOI: 10.1103/PhysRevB.80.224104

PACS number(s): 61.72.jd, 61.72.Bb, 71.15.Pd

\section{INTRODUCTION}

Metals in body-centered cubic (bcc) crystal structure, e.g., Mo, Ta, and $\mathrm{W}$, have long posed a serious challenge for computational materials science. Thermophysical properties such as the specific heat show unusually large anharmonic contributions, and until recently, electronic-structure calculations were in stark qualitative and quantitative disagreement with experiments, e.g., concerning the relative stability between competing phases. ${ }^{1,2}$ Turning to lattice defects in bcc metals, a similarly involved situation exists. For instance, the core structure of screw dislocations, which governs the resistance to plastic deformation, is notoriously difficult to model. ${ }^{3}$ Even when considering the simplest lattice defect, the vacancy, very large uncertainties persist. ${ }^{4}$ Since vacancies, among others, govern substitutional diffusion in crystals, the lack of quantitative understanding of their properties impedes the development of a theory of diffusion in bcc metals and alloys similar to the one that has been established in face-centered cubic systems such as Al. ${ }^{5,6}$

In this paper, we focus on vacancy formation and migration in Mo. Experimentally, although the self-diffusion rate is well known, ${ }^{4}$ quantities related to vacancies show very large scatter. Values of the vacancy-formation energy are either in the range $2.6-3.2 \mathrm{eV}$ or $3.4-3.8 \mathrm{eV},{ }^{4,7}$ and estimates of the vacancy concentration close to the melting point differ by many orders of magnitude. ${ }^{8}$ We present a microscopic model of vacancy formation and self-diffusion in Mo in quantitative agreement with experimental self-diffusion data over a wide range of temperatures. Our modeling is based on large-scale density-functional theory (DFT) molecular-dynamics (MD) simulations and the use of a recently developed exchangecorrelation functional [Armiento-Mattsson 2005 (AM05)], ${ }^{9}$ and it involves no experimental parameters. The current results resolve many ambiguities related to vacancies and selfdiffusion in Mo, and thus opens for a type of microscopic modeling that previously has been unavailable for bcc metals and alloys.

\section{SELF-DIFFUSION AS A THERMALLY ACTIVATED PROCESS}

A common characteristic of bcc metals is an increase with temperature of the apparent activation energy $Q$ for selfdiffusion, leading to a nonlinear relation in an Arrhenius plot of the diffusion rate. ${ }^{4}$ This anomaly has historically been attributed to the onset of some additional diffusion mechanism contributing at high temperature. However, all such ad hoc assumptions have been ruled out, ${ }^{10}$ and it is now believed that the vacancy mechanism alone is responsible for self-diffusion in bcc metals, as it is in fcc metals. The selfdiffusion rate $D$ then depends on the vacancy concentration $c_{\mathrm{v}}$ and the vacancy migration rate $\Gamma_{\mathrm{v}}$ as

$$
D=\frac{1}{6} f l^{2} c_{\mathrm{v}} \Gamma_{\mathrm{v}},
$$

where $f$ is a geometrical factor (the correlation factor, $f$ $=0.73$ in a bcc crystal) and $l$ is the jump length in the crystal.

A microscopic model of diffusion in bcc metals needs to provide $c_{\mathrm{v}}$ and $\Gamma_{\mathrm{v}}$ as a function of temperature. Alternatively, the model can instead yield the entropy $S_{\mathrm{v}}^{\mathrm{f}}$ and enthalpy $H_{\mathrm{v}}^{\mathrm{f}}$ of vacancy formation, and the corresponding quantities governing vacancy migration, $S_{\mathrm{v}}^{\mathrm{m}}$ and $H_{\mathrm{v}}^{\mathrm{m}}$, since both these processes are thermally activated,

$$
\begin{gathered}
c_{\mathrm{v}}=\exp \left(S_{\mathrm{v}}^{\mathrm{f}} / k_{\mathrm{B}}-H_{\mathrm{v}}^{\mathrm{f}} / k_{\mathrm{B}} T\right) \\
\Gamma_{\mathrm{v}}=\Gamma_{0} \exp \left(S_{\mathrm{v}}^{\mathrm{m}} / k_{\mathrm{B}}-H_{\mathrm{v}}^{\mathrm{m}} / k_{\mathrm{B}} T\right) .
\end{gathered}
$$

Here, $\Gamma_{0}$ is a trivial prefactor within the transition-state theory (TST) (Ref. 11) and $k_{\mathrm{B}} T$ is Bolzmann's constant times 
temperature. The temperature variation in $Q$ therefore corresponds to temperature variation in $H_{\mathrm{v}}^{\mathrm{f}}$ and/or in $H_{\mathrm{v}}^{\mathrm{m}}$, both possibilities have been suggested in the literature. ${ }^{12,13}$

In order to resolve the origin of the anomalous selfdiffusion rate in bcc metals, we employ extensive DFT (Refs. 14 and 15) MD simulations to calculate (i) the temperature variation in $H_{\mathrm{v}}^{\mathrm{f}}$ and (ii) the absolute jump rate $\Gamma_{\mathrm{v}}$ of vacancies at high temperature. In combination with established methods for calculating the limiting low-temperature (classical) values of $H_{\mathrm{v}}^{\mathrm{f}}, S_{\mathrm{v}}^{\mathrm{f}}, H_{\mathrm{v}}^{\mathrm{m}}$, and $S_{\mathrm{v}}^{\mathrm{m}}$, this allows for a full microscopic description of vacancy formation and selfdiffusion in Mo.

\section{APPLYING DENSITY-FUNCTIONAL THEORY TO CALCULATE DEFECT ENERGIES}

The accuracy of a DFT calculation is determined by the exchange-correlation term used. ${ }^{16}$ We choose the recently developed AM05 functional. ${ }^{9,17}$ It has demonstrated high fidelity for transition metals, refractory metals, semiconductors, and oxides. ${ }^{18}$ For Mo, application of the AM05 functional yields a $0 \mathrm{~K}$ lattice constant of $3.134 \AA$ and a bulk modulus of $283 \mathrm{GPa}$. Particularly important for studies of defects, AM05 includes the intrinsic surface error. ${ }^{19,20}$ Earlier functionals require postprocessing corrections to be made when analyzing vacancy energies, ${ }^{19,20}$ corrections that cannot be made during a DFT-MD simulation. Taken together, the beneficial properties of AM05 make it meaningful to attempt the computationally demanding DFT-MD runs required for the current problem.

The DFT-MD simulations were performed with a massively parallel version of the PAW core-potential code VASP 5.1.40 (Refs. 21-25) on Cray-XT (Ref. 26) using stringent convergence settings. ${ }^{16}$ We used a plane-wave cutoff of 400 eV using the 6 electron Mo PAW_PBE core function of 08Apr2002. Real-space projections are not applied, to maintain highest possible accuracy. All results presented are for the mean-value $k$ point $(1 / 4,1 / 4,1 / 4)$. Convergence with respect to $k$ points was investigated by comparing energy differences between $\Gamma$ point $(0,0,0)$, mean-value point $(1 / 4,1 /$ $4,1 / 4)$, and Monkhorst-Pack grids $\left(2^{3}\right.$ and $\left.4^{3}\right)$. Using the mean-value point is a significant improvement upon the $\Gamma$ point, with little to no improvement when going to higher grids. The electronic states are distributed according to Mermin's finite-temperature formulation of DFT. ${ }^{27}$

Structural optimization was used to find the lowtemperature limit of the vacancy-formation energy $H_{\mathrm{v}}^{\mathrm{f}}$ and the migration activation energy $H_{\mathrm{v}}^{\mathrm{m}}$ in 128 atom supercells. The vacancy formation entropy $S_{\mathrm{v}}^{\mathrm{f}}$ and the migration prefactor $\Gamma_{0} \exp \left(S_{\mathrm{v}}^{\mathrm{m}} / k_{\mathrm{B}} T\right)$ were obtained by calculating force constant matrices ${ }^{5}$ via finite displacements, ${ }^{28}$ this was done by calculating the force-constant matrix via finite displacements $^{5}$ in 54 atom unit cells. To verify that the size of the system is adequate, we performed separate model potential calculations from 54 to 800 atoms. We find that prefactors calculated via the force-constant matrix method are well represented in the 54-atoms supercells. The DFT-MD simulations, which are necessary for the study of the anharmonic contributions to the formation and migration free en-

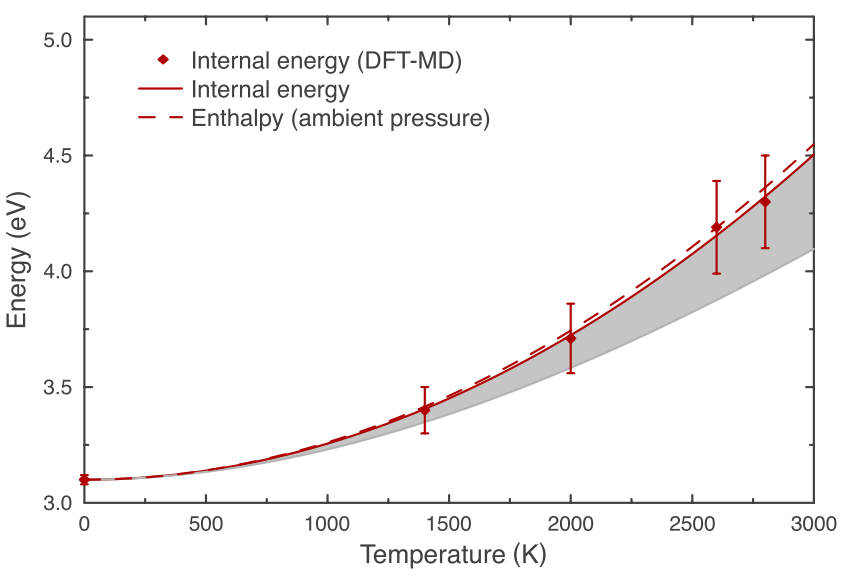

FIG. 1. (Color online) The formation energy of a vacancy in Mo, as a function of temperature. The error bars are based on the fluctuations in the DFT-MD calculations and correspond to two standard deviations. The statistical inefficiency was determined using block averages. The major part of the temperature dependence in $H_{\mathrm{v}}^{\mathrm{f}}$ comes from anharmonic lattice vibrations. The shaded area illustrates the enthalpy contribution due to thermal effects in the occupation of electronic states, see text.

ergies, are the most computationally demanding parts of the present work.

\section{VACANCY FORMATION ENERGY FROM MD SIMULATIONS}

The vacancy-formation energy was obtained by comparing thermally averaged energies in a system containing a vacancy, with that in a bulk system, using DFT-MD. The simulations were done at fixed volumes $V$, corresponding to the relaxed volumes of the bulk and vacancy systems at zero kelvin, respectively. With increasing temperature the pressure therefore increases by $\Delta p(T)$. The corresponding increase in free energy is to lowest order $\Delta G(T)$ $=V \Delta p(T)^{2} /(2 \mathrm{~K})$. We subtract this term, calculated separately for the bulk and vacancy systems, by using the pressure from the DFT-MD calculations, and bulk moduli $K$ from static DFT calculations. The corresponding change in predicted vacancy concentration is relatively small, which can be understood in the following way. The increase with temperature, $\Delta p(T)$, is somewhat larger in the bulk system compared to the vacancy system, but it is weighted by a factor $127 / 128$ in the calculation of the change in free energy per vacancy. The net effect is that the predicted vacancy concentration is changed by less than $20 \%$. The bulk modulus of the vacancy system is lower than that of the bulk system by approximately $2.5 \%$, which is roughly in line with the expectation that $K$ varies as the square of the electronic density. ${ }^{29}$

Figure 1 shows the considerable anharmonic increase in $H_{\mathrm{v}}^{\mathrm{f}}$ with temperature. The increase is well described by a quadratic polynomial in $T$. By using the thermodynamic relation $T \frac{\partial S}{\partial T}=\frac{\partial H}{\partial T}, S_{\mathrm{v}}^{\mathrm{f}}$ can be obtained, with the reference formation entropy $S_{\mathrm{v}}^{\mathrm{f}}(T=0)$ taken from phonon calculations. Table I presents the calculated vacancy-formation parameters, and 
TABLE I. Calculated vacancy formation and migration parameters compared with experimental data. Static DFT means classical quantities at $0 \mathrm{~K}$. DFT-MD includes thermal effects of vibrational and electronic origin. For each experimental value, we have compared with DFT-MD results for the same temperature (interval) as indicated below.

\begin{tabular}{lcccccc}
\hline \hline & $\begin{array}{c}H_{\mathrm{v}}^{\mathrm{f}} \\
(\mathrm{eV})\end{array}$ & $\begin{array}{c}H_{\mathrm{v}}^{\mathrm{m}} \\
(\mathrm{eV})\end{array}$ & $\begin{array}{c}Q \\
(\mathrm{eV})\end{array}$ & $S_{\mathrm{v}}^{\mathrm{f}}\left(k_{\mathrm{B}}\right)$ & $\begin{array}{c}\Gamma_{0} e^{S_{\mathrm{v}}^{\mathrm{m}} / k_{\mathrm{B}}} \\
(\mathrm{Hz})\end{array}$ & $\begin{array}{c}c_{\mathrm{v}}\left(T_{\text {melt }}\right) \\
(\%)\end{array}$ \\
\hline Static DFT & 3.10 & 1.30 & 4.40 & 0.7 & $1.5 \times 10^{13}$ & 0.18 \\
DFT-MD & 3.90 & 1.32 & 5.04 & 2.30 & $10^{-3}, \ldots, 10^{\mathrm{c}}$ \\
Experiments & $3.0 \pm 0.2^{\mathrm{a}}$ & $1.30^{\mathrm{a}}$ & $4.78^{\mathrm{b}}$ & & 2890 \\
& $3.6 \pm 0.2^{\mathrm{d}}$ & & & & $1.500,2000$ & $2000, \ldots, 2500$ \\
Temperature $(\mathrm{K})$ & $2000, \ldots, 2500$ & $560, \ldots, 640$ & $1350, \ldots, 2000$ \\
\hline \hline
\end{tabular}

${ }^{\text {aReference } 4 .}$

${ }^{\mathrm{b}}$ Reference 34

${ }^{\mathrm{c}}$ Reference 8 .

${ }^{\mathrm{d}}$ Reference 7 (positron lifetime spectroscopy data).

the vacancy concentration at the melting temperature $T_{\mathrm{m}}$, along with available experimental data.

The broadening of the Fermi-Dirac distribution $f$ of electronic states occurring at finite temperature is naturally taken into account in the MD simulations, within the DFT. For a given density of states $N$, one can express the electronic entropy as ${ }^{30}$

$$
S_{\mathrm{el}}=-2 k_{\mathrm{B}} \int_{-\infty}^{\infty}[f(\ln f)+(1-f) \ln (1-f)] N d E,
$$

where the integration is over energy, $E$. Via thermodynamic integration one obtains the corresponding shift in inner energy. This is shown as the shaded region in Fig. 1, illustrating the relative effect of the broadening of the Fermi-Dirac function and of anharmonic lattice vibrations. However, it should be noted that the two are coupled, via the minimization of the total free energy.

\section{DIRECT SIMULATION OF VACANCY MOBILITY}

In the simulation of a single vacancy in a 128 lattice-point supercell at $T=2600$ and $2800 \mathrm{~K}$, the vacancy will migrate by thermal activation. This allows us to directly estimate the mean jump rate, $\Gamma_{\mathrm{v}}$, without the additional assumptions and approximations of ${ }^{11}$ TST. In order to unambiguously locate the vacancy at each time step, we use a model potential ${ }^{31}$ to quench a copy of each atomic configuration, and associate the empty lattice site with the position of the vacancy ${ }^{32}$ hereby obtaining a trajectory in time of the vacancy migration. In the 2600 and $2800 \mathrm{~K}$ simulations, we logged 8 and 11 jumps, respectively, with the simulation at $2600 \mathrm{~K}$ being longer in real time. The resulting rate is shown in Fig. 2.

Based on the direct estimate of the jump rate at 2600 and $2800 \mathrm{~K}$, and the calculation of $c_{\mathrm{v}}$, we next compare theoretical and experimental diffusion rates at high $T$, see Fig. 3. Assuming that $H_{\mathrm{v}}^{\mathrm{m}}$ has a quadratic temperature variation, like $H_{\mathrm{v}}^{\mathrm{f}}$, we can combine the low-temperature harmonic TST estimate of $\Gamma_{\mathrm{v}}$ with the high-temperature rates, and thereby obtain jump rates over the full experimental range.
By comparing long model potential simulations in 127-, 1023-, and 3455-atom systems, we conclude that the jump rate in a 127 -atom system is representative of that in larger systems to within $5 \%$. We make the following observations: the major part of the temperature dependence in the diffusion activation energy $Q$ can be attributed to vacancy formation, with a smaller but significant part associated with vacancy migration. The AM05 results can be considered in quantitative agreement with experimental data when considering the difficulties involved in accurate calculations of point defects and point-defect kinetics in transition metals. ${ }^{19}$ There is a significant difference between using the generalized gradient approximation by Perdew, Burke, and Ernzerhof ${ }^{33}$ and AM05, see Fig. 3, affirming the central importance of the exchange-correlation functional also for solid-state systems. ${ }^{18,19}$ The $0 \mathrm{~K}$ vacancy-formation energy is $2.67 \mathrm{eV}$ in PBE. ${ }^{19}$ Full DFT-MD simulations, using PBE, were performed to obtain the temperature dependence of the vacancy-

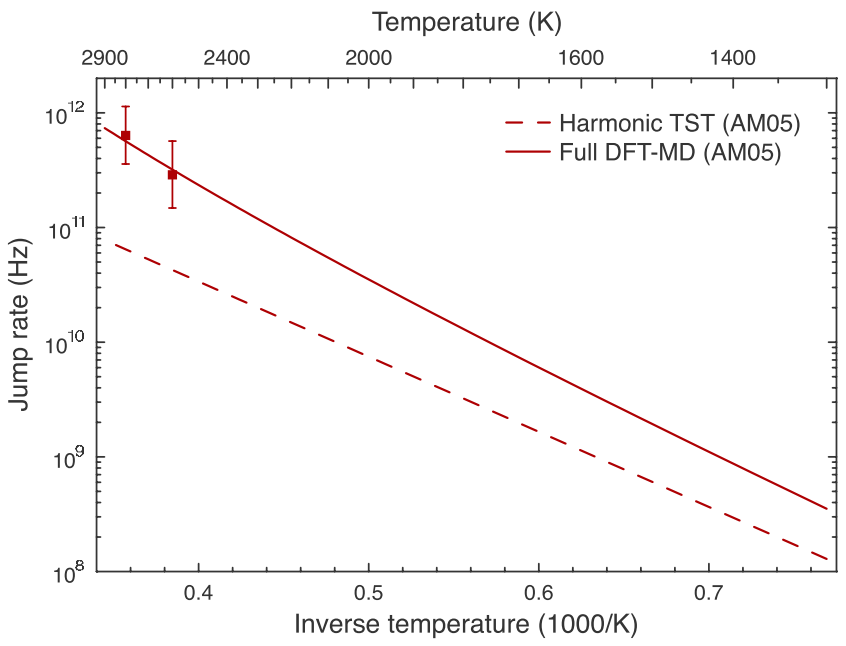

FIG. 2. (Color online) Calculated vacancy jump rates. Squares show direct jump rates obtained in first-principles MD simulations, while the dashed line represents the harmonic TST result. The solid line was drawn under the assumption that the activation energy for vacancy migration varies quadratically with temperature, see text. 


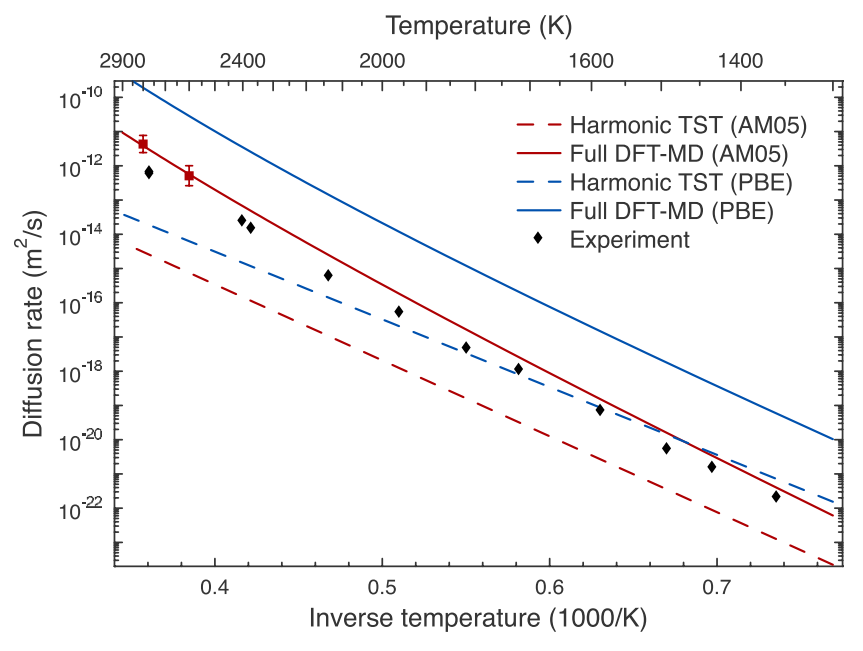

FIG. 3. (Color online) Self-diffusion rates in Mo calculated with DFT-MD compared to experiments (Ref. 34). The main calculation uses the AM05 functional, results for PBE are included for comparison. The error bars correspond to two standard deviations in the estimation of the mean jump rate, which is the dominating source of error.

formation enthalpy. For vacancy migration, the temperature dependence was approximated with the one of AM05.

The effective activation energy $Q$ is $4.78 \mathrm{eV}$ in experiments from a low-temperature fit $(1350<T<2000 \mathrm{~K})$, compared to $5.0 \mathrm{eV}$ in our AM05 calculations. The corresponding energies, at high $T(T>2000 \mathrm{~K})$, are $5.50 \mathrm{eV}$ in experiments and $5.78 \mathrm{eV}$ for AM05. Thus, there is a consistent overestimation of $Q=H_{\mathrm{v}}^{\mathrm{f}}+H_{\mathrm{v}}^{\mathrm{m}}$ by $0.2-0.3 \mathrm{eV}$, or only about $5 \%$. It is important to note that the simulations have no free parameters and hence can be repeated for materials and conditions where experimental data is nonexistent, scarce, expensive to obtain, or disputed. We conclude that the systematic high fidelity of AM05 for bulk properties ${ }^{18}$ translates into accurate results also for self-diffusion, a significantly more complex property.

We now discuss the current results for vacancies in the light of available experimental information. Data on vacancies in Mo are mainly from two types of experiments, ${ }^{4}$ resistivity measurements after quenching, ${ }^{35}$ and positron annihilation experiments. ${ }^{7}$ Both refer to temperatures above 2000 $\mathrm{K}$. Resistivity measurements are, in principle, very accurate, but the kinetic loss of vacancies during quenching must be accounted for. Typically, this is done by extrapolating vacancy mobility data from $\approx 600 \mathrm{~K}$ up to $2000-2500 \mathrm{~K}$. With our current estimate of the vacancy mobility, we find that the quenching loss has been underestimated in previous analyses, leading to an underestimation also of the effective vacancy-formation enthalpy at high $T$. We have reanalyzed resistivity data in Ref. 35 based on the current calculated vacancy mobility. The resulting $H_{\mathrm{v}}^{\mathrm{f}}=3.65 \mathrm{eV}$ rather than the previously estimated $3.2 \mathrm{eV}$ (Ref. 35) at $T=2000-2500 \mathrm{~K}$.

From resistivity data, the absolute vacancy concentration at $T_{\mathrm{m}}$ may be obtained if the specific resistivity $\rho_{\mathrm{v}}$ (per vacancy) is known. A typical estimate is ${ }^{35} \rho_{\mathrm{v}}=4.33$ $\times 10^{-6} \Omega \mathrm{m}$, which leads to $c_{\mathrm{v}}=0.0013 \%$ in the analysis of Ref. 35 or $c_{\mathrm{v}}=0.005 \%$ in the above analysis. However, this

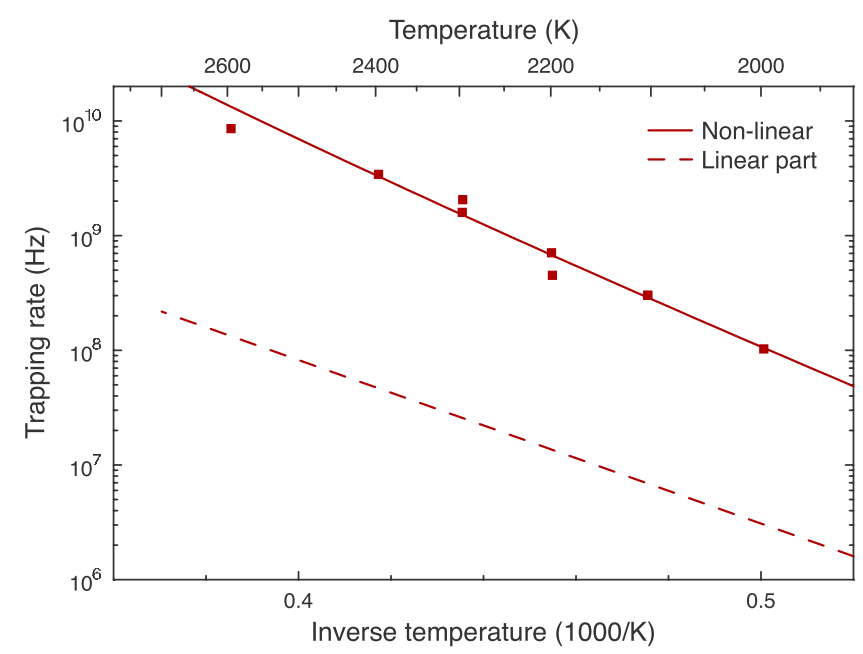

FIG. 4. (Color online) Positron trapping rate $\sigma c_{\mathrm{v}}$. The full line is fitted to experimental data (Ref. 36) assuming a temperature variation in $H_{\mathrm{v}}^{\mathrm{f}}$ as in Fig. 1. The dashed line is the corresponding linear term.

value of $\rho_{\mathrm{v}}$ is probably too high, since if $c_{\mathrm{v}}\left(T_{\mathrm{m}}\right)=0.001 \%$, then, the experimental diffusion rate in combination with Eq. (1) leads to a very high jump rate, $\Gamma\left(T_{\mathrm{m}}\right)=22 \mathrm{THz}$, which is much higher than the cut-off frequency in the phonon spectrum.

Positron annihilation experiments are done under equilibrium conditions, as opposed to resistivity measurements. In such an experiment, either the Doppler-broadening, lifetime $(\bar{\tau})$, or positron lifetime spectrum (PLS) of annihilating positrons are measured. To distinguish trapped positrons from free (bulk) positrons, a model of positron trapping is applied in which assumptions are needed about the temperature dependencies of lifetimes and/or trapping rates. From PLS data one can derive the trapping rate $\sigma c_{\mathrm{v}}$, where $\sigma$ is the trapping rate per vacancy, without further assumptions about lifetimes. We fitted data from Ref. 7 by using the quadratic temperature dependence of $H_{\mathrm{v}}^{\mathrm{f}}$ to fit $\sigma c_{\mathrm{v}}$, see Fig. 4. The resulting $0 \mathrm{~K}$ formation energy $H_{\mathrm{v}}^{\mathrm{f}}(T=0)=2.85 \mathrm{eV}$, which is slightly below our calculated $3.10 \mathrm{eV}$. However, this result assumes that the temperature variation in $\sigma$ can be neglected.

Absolute vacancy concentrations may now be obtained if the specific trapping rate $\sigma$ is known. There are no estimates of $\sigma$ in Mo, but from the fit one obtains a value of $\sigma \exp \left(S_{\mathrm{v}}^{\mathrm{f}} / k_{\mathrm{B}}\right)$, which in our case amounts to $0.5 \times 10^{14} \mathrm{~Hz}$. This value compares reasonably well with that in $\mathrm{Al},{ }^{7}$ where the vacancy concentration has been independently measured.

\section{SUMMARY}

We have presented and validated a microscopic modeling approach based on DFT-MD that describes the anharmonic behavior of Mo vacancy diffusion at high temperature and can be applied to a wide range of materials. The simulations provide a quantitative microscopic model of vacancy motion: temperature dependence of the vacancy-formation energy, absolute concentration, and vacancy jump rate. The successful results for defects and diffusion in Mo opens for a type of microscopic modeling that previously have been unavailable 
for bcc metals and alloys. We therefore expect the current results to stimulate further work on defects and diffusion in bcc systems.

\section{ACKNOWLEDGMENTS}

We thank Göran Grimvall for discussions and Odd Runevall for doing some of the verification calculations. We also thank Georg Kresse for the early opportunity to employ VASP 5.1 and Paul Kent for sharing code modifications for the
Cray XT4 platform Red Storm at Sandia High Performance Computing. R.A. gratefully acknowledges support from the Alexander von Humboldt Foundation. The work was supported by the NNSA Science Campaigns (T.R.M.) and the Advanced Simulation \& Computing Campaign (A.E.M.) at Sandia National Laboratories. Sandia is a multiprogram laboratory operated by Sandia Corporation, a Lockheed Martin Co., for the United States Department of Energy's National Nuclear Security Administration under Contract No. DE-AC04-94AL85000.
${ }^{1}$ C. Asker, A. B. Belonoshko, A. S. Mikhaylushkin, and I. A. Abrikosov, Phys. Rev. B 77, 220102(R) (2008).

${ }^{2}$ V. Ozoliňš, Phys. Rev. Lett. 102, 065702 (2009).

${ }^{3}$ T. E. Jones, M. E. Eberhart, D. P. Clougherty, and C. Woodward, Phys. Rev. Lett. 101, 085505 (2008).

${ }^{4} \mathrm{H}$. Schultz, in Atomic Defects in Metals, Landolt-Börnstein New Series Vol. III/25, edited by H. Ullmaier (Springer-Verlag, Berlin, 1991).

${ }^{5}$ M. Mantina, Y. Wang, R. Arroyave, L. Q. Chen, Z. K. Liu, and C. Wolverton, Phys. Rev. Lett. 100, 215901 (2008).

${ }^{6}$ D. Simonovic and M. H. F. Sluiter, Phys. Rev. B 79, 054304 (2009).

${ }^{7}$ H.-E. Schaefer, Phys. Status Solidi A 102, 47 (1987).

${ }^{8}$ A. Choudhury and C. R. Brooks, Int. J. Thermophys. 5, 403 (1984).

${ }^{9}$ R. Armiento and A. E. Mattsson, Phys. Rev. B 72, 085108 (2005).

${ }^{10} \mathrm{~J}$. L. Bocquet, G. Brebec, and Y. Limoge, in Diffusion in Metals and Alloys, 4th ed., edited by R. W. Cahn and P. Haasen (NorthHolland, Amsterdam, 1996).

${ }^{11}$ G. H. Vineyard, J. Phys. Chem. Solids 3, 121 (1957).

${ }^{12}$ H. R. Schober, W. Petry, and J. Trampenau, J. Phys.: Condens. Matter 4, 9321 (1992).

${ }^{13}$ A. Satta, F. Willaime, and S. de Gironcoli, Phys. Rev. B 57, 11184 (1998).

${ }^{14}$ P. Hohenberg and W. Kohn, Phys. Rev. 136, B864 (1964).

${ }^{15}$ W. Kohn and L. J. Sham, Phys. Rev. 140, A1133 (1965).

${ }^{16}$ A. E. Mattsson, Peter A. Schultz, Michael P. Desjarlais, Thomas R. Mattsson, and Kevin Leung, Modell. Simul. Mater. Sci. Eng. 13, R1 (2005).
${ }^{17}$ W. Kohn and A. E. Mattsson, Phys. Rev. Lett. 81, 3487 (1998).

${ }^{18}$ A. E. Mattsson, Rickard Armiento, Joachim Paier, Georg Kresse, John M. Wills, and Thomas R. Mattsson, J. Chem. Phys. 128, 084714 (2008).

${ }^{19}$ T. R. Mattsson and A. E. Mattsson, Phys. Rev. B 66, 214110 (2002).

${ }^{20}$ K. Carling, G. Wahnstrom, T. R. Mattsson, A. E. Mattsson, N. Sandberg, and G. Grimvall, Phys. Rev. Lett. 85, 3862 (2000).

${ }^{21}$ G. Kresse and J. Hafner, Phys. Rev. B 47, 558 (1993).

${ }^{22}$ G. Kresse and J. Hafner, Phys. Rev. B 49, 14251 (1994).

${ }^{23}$ G. Kresse and J. Furthmüller, Phys. Rev. B 54, 11169 (1996).

${ }^{24}$ P. E. Blöchl, Phys. Rev. B 50, 17953 (1994).

${ }^{25}$ G. Kresse and D. Joubert, Phys. Rev. B 59, 1758 (1999).

${ }^{26}$ P. R. C. Kent, J. Phys.: Conf. Ser. 125, 012058 (2008).

${ }^{27}$ N. D. Mermin, Phys. Rev. 137, A1441 (1965).

${ }^{28}$ V. Ozoliņš, B. Sadigh, and M. Asta, J. Phys.: Condens. Matter 17, 2197 (2005).

${ }^{29} \mathrm{C}$. $\mathrm{Li}$ and P. Wu, Chem. Mater. 13, 4642 (2001).

${ }^{30} \mathrm{G}$. Grimvall, Thermophysical Properties of Materials (Elsevier, Amsterdam, 1999).

${ }^{31}$ M. W. Finnis and J. E. Sinclair, Philos. Mag. A 50, 45 (1984).

${ }^{32}$ N. Sandberg, B. Magyari-Kope, and T. R. Mattsson, Phys. Rev. Lett. 89, 065901 (2002).

${ }^{33}$ J. P. Perdew, K. Burke, and M. Ernzerhof, Phys. Rev. Lett. 77, 3865 (1996).

${ }^{34}$ K. Maier, H. Mehrer, and G. Rein, Z. Metallkd. 70, 271 (1979).

${ }^{35}$ I. A. Schwirtlich and H. Schultz, Philos. Mag. A 42, 601 (1980).

${ }^{36}$ R. Ziegler and H. E. Schaefer, Mater. Sci. Forum 15-18, 145 (1987). 